\title{
A comparison of spinal laser interstitial thermotherapy with open surgery for metastatic thoracic epidural spinal cord compression
}

\author{
Dhiego Chaves de Almeida Bastos, MD, ${ }^{1}$ Richard George Everson, MD, ${ }^{2}$ \\ Bruno Fernandes de Oliveira Santos, MD, ${ }^{1}$ Ahmed Habib, MD, ${ }^{1}$ Rafael A. Vega, MD, PhD, ${ }^{1}$ \\ Marilou Oro, DNP, Ganesh Rao, MD, ${ }^{1}$ Jing Li, MD, ${ }^{3}$ Amol J. Ghia, MD, ${ }^{3}$ Andrew J. Bishop, MD, ${ }^{3}$ \\ Debra Nana Yeboa, MD, ${ }^{3}$ Behrang Amini, MD, PhD, ${ }^{4}$ Laurence D. Rhines, MD, ${ }^{1}$ and \\ Claudio Esteves Tatsui, MD1
}

\begin{abstract}
Departments of ${ }^{1}$ Neurosurgery, ${ }^{3}$ Radiation Oncology, and ${ }^{4}$ Diagnostic Radiology, The University of Texas MD Anderson Cancer Center, Houston, Texas; and 'Department of Neurosurgery, David Geffen School of Medicine at UCLA, Los Angeles, California
\end{abstract}

\begin{abstract}
OBJECTIVE The proximity of the spinal cord to compressive metastatic lesions limits radiosurgical dosing. Open surgery is used to create safe margins around the spinal cord prior to spinal stereotactic radiosurgery (SSRS) but carries the risk of potential surgical morbidity and interruption of systemic oncological treatment. Spinal laser interstitial thermotherapy (SLITT) in conjunction with SSRS provides local control with less morbidity and a shorter interval to resume systemic treatment. The authors present a comparison between SLITT and open surgery in patients with metastatic thoracic epidural spinal cord compression to determine the advantages and disadvantages of each method.
\end{abstract}

METHODS This is a matched-group design study comprising patients from a single institution with metastatic thoracic epidural spinal cord compression that was treated either with SLITT or open surgery. The two cohorts defined by the surgical treatment comprised patients with epidural spinal cord compression (ESCC) scores of 1c or higher and were deemed suitable for either treatment. Demographics, pre- and postoperative ESCC scores, histology, morbidity, hospital length of stay (LOS), complications, time to radiotherapy, time to resume systemic therapy, progression-free survival (PFS), and overall survival (OS) were compared between groups.

RESULTS Eighty patients were included in this analysis, 40 in each group. Patients were treated between January 2010 and December 2016. There was no significant difference in demographics or clinical characteristics between the cohorts. The SLITT cohort had a smaller postoperative decrease in the extent of ESCC but a lower estimated blood loss (117 vs $1331 \mathrm{ml}, p<0.001)$, shorter LOS (3.4 vs 9 days, $p<0.001)$, lower overall complication rate $(5 \%$ vs $35 \%, p$ $=0.003$ ), fewer days until radiotherapy or SSRS (7.8 vs 35.9, $p<0.001)$, and systemic treatment ( 24.7 vs 59 days, $p=$ 0.015 ). PFS and $O S$ were similar between groups ( $p=0.510$ and $p=0.868$, respectively).

CONCLUSIONS The authors' results have shown that SLITT plus XRT is not inferior to open decompression surgery plus XRT in regard to local control, with a lower rate of complications and faster resumption of oncological treatment. A prospective randomized controlled study is needed to compare SLITT with open decompressive surgery for ESCC. https://thejns.org/doi/abs/10.3171/2019.10.SPINE19998

KEYWORDS epidural spinal cord compression; laser interstitial thermal therapy; separation surgery; spinal metastases; spinal stereotactic radiosurgery; thoracic spine; oncology; surgical technique

$\mathrm{T}$ HE role of surgery in the management of spinal metastatic disease continues to evolve, especially regarding its pairing with recent advances in systemic treatment and radiotherapy. Improved functional outcomes have been demonstrated with decompressive surgery and spinal stabilization followed by conventional external-beam radiation therapy (cEBRT) over cEBRT alone in patients with symptomatic epidural spinal cord compression (ESCC). ${ }^{12}$ More recently, spinal stereotactic radiosurgery (SSRS) has emerged as a highly effective

ABBREVIATIONS cEBRT = conventional external-beam radiation therapy; EBL = estimated blood loss; ESCC = epidural spinal cord compression; iMRI = intraoperative MRI; KPS = Karnofsky Performance Scale; LOS = length of stay; OS = overall survival; PFS = progression-free survival; SLITT = spinal laser interstitial thermotherapy; SSRS = spinal stereotactic radiosurgery.

SUBMITTED August 26, 2019. ACCEPTED October 11, 2019.

INCLUDE WHEN CITING Published online January 3, 2020; DOI: 10.3171/2019.10.SPINE19998. 
treatment, overcoming the traditional resistance of certain tumor types to cEBRT, and providing exceptional rates of tumor control. $3,7,20$

However, in cases of high-grade ESCC, proximity to the spinal cord results in reduced radiation doses to the epidural space in order to minimize toxicity. In such cases, Bilsky et al., recommended a more limited surgical decompression aimed at reconstituting the subarachnoid space around the spinal cord (separation surgery) to allow a margin for the safe delivery of a cytotoxic dose of radiation to the remaining tumor while respecting the spinal cord toxicity constraints. ${ }^{11}$ This approach combined with subsequent SSRS has been shown to provide excellent local tumor control. ${ }^{10}$ Although considered a less invasive approach, separation surgery still poses a heavy postoperative burden for patients who may be debilitated, and is associated with a delay in the administration of systemic therapy. ${ }^{6,19,21}$

Our group previously reported the use of spinal laser interstitial thermotherapy (SLITT) as a less invasive alternative to separation surgery to create a safe margin for the delivery of SSRS..$^{15}$ SLITT involves placement of a laser probe directly into the epidural tumor close $(5-6 \mathrm{~mm})$ to the dural margin. ${ }^{16}$ The laser ablation of the tumor is monitored in real time with MR thermography to protect the spinal cord from thermal damage. This procedure, as with separation surgery, is ideally followed by SSRS in standard cytotoxic doses to cover the gross tumor volume. ${ }^{8}$ If spinal instability is suspected, one can perform percutaneous placement of spinal instrumentation and/or methylmethacrylate augmentation at the same time or in a second stage. ${ }^{14}$

Here, we report a retrospective comparison between two cohorts of patients with ESCC due to metastatic disease. The first cohort comprised patients who underwent SLITT followed by adjuvant radiotherapy. The second cohort comprised patients treated with standard open surgical decompression, followed by adjuvant radiotherapy. The objective of the study was to compare the outcomes for the groups, as well as complication rates.

\section{Methods \\ Study Design}

This is a matched-group study comparing 2 groups of patients with thoracic spine epidural metastases treated with different surgical modalities, between January 2010 and December 2016. The first group comprised consecutive patients treated with SLITT, and the second group comprised patients treated with open surgical decompression. The clinical records of patients with thoracic spinal epidural metastases were retrospectively reviewed. Inclusion criteria were 1) preoperative MRI demonstrating epidural spinal cord compression arising from a tumor located in a vertebral body between T2 and T12, with a Bilsky score of $1 \mathrm{c}, 2$, or $3 ;{ }^{2}$ ) epidural tumor contained within the boundaries of the posterior longitudinal ligament or periosteum of the dorsal elements; and 3) suitable candidate for either treatment modality through a careful analysis performed by the senior author. Patients presenting with severe neurological deficits (Frankel grade A, B, or C) were not included in the comparison nor were those who could not undergo MRI (e.g., because of a pacemaker). The groups were matched based on variables that could correlate with local recurrence and/or overall survival. The variables selected were 1) Bilsky score, 2) Karnofsky Performance Scale (KPS) score, 3) age, 4) prior radiation treatment, and 5) adjuvant radiation treatment.

Charts and images were reviewed to evaluate patient demographics, tumor histology, site, degree of pre- and postoperative epidural tumor compression (using the Bilsky ESCC grading scale ${ }^{2}$ ), complications, and treatmentrelated factors. The interval from prior radiotherapy, the interval to initiation of any type of adjuvant treatment, hospital length of stay (LOS), estimated blood loss (EBL), and surgical mortality and morbidity were recorded. Motor function was evaluated according to the Frankel classification. ${ }^{5}$ Progression-free survival (PFS) was defined as the time between the procedure and local recurrence or last follow-up. Overall survival (OS) was defined as the time interval between the procedure and patient's death or censored. Complications were defined as any adverse event within 30 days related to the procedure. Major complications were defined as medical or surgical complications that required a prolonged hospital stay or new surgical procedure. Complications related to each procedure were recorded and were deemed either minor or major complications (for a list of all procedure-related complications, see Supplemental Table 1). The institutional review board approved this study, and, due to its retrospective nature, patient consent was waived.

\section{Open Surgery}

All patients underwent circumferential decompression via a standard posterior approach, which included a laminectomy, unilateral or bilateral facetectomies, and circumferential resection of the epidural tumor. Pedicle screw-rod constructs were used in the setting of instability. Vertebral body and additional soft-tissue resections were performed depending on the location of the tumor and at the discretion of the surgeon. When necessary, anterior column reconstruction was performed using either polymethylmethacrylate or an expandable cage.

\section{Spinal Laser Interstitial Thermotherapy}

SLITT has been previously described. ${ }^{17}$ Briefly, the patient is positioned prone, with the arms parallel to the body on the intraoperative MRI (iMRI) transfer table. Fiducial markers (IZI Medical Products) are randomly placed in the dorsal region overlying the area of interest, and the patient is transferred to the iMRI unit where T2weighted images of the region of interest are obtained, uploaded to the navigation software (Medtronic, Inc.), and used for surface-matching image guidance registration. Image guidance is used to advance a navigated Jamshidi needle (Medtronic Inc.) to the final target at a distance of 5-6 $\mathrm{mm}$ from the dural edge. We estimated that each laser fiber can achieve a 10 -mm-diameter sphere of thermal damage; therefore, multiple needles may be positioned in tandem to treat larger tumors. A K-wire is used to exchange the Jamshidi needle for a plastic access cannula, 

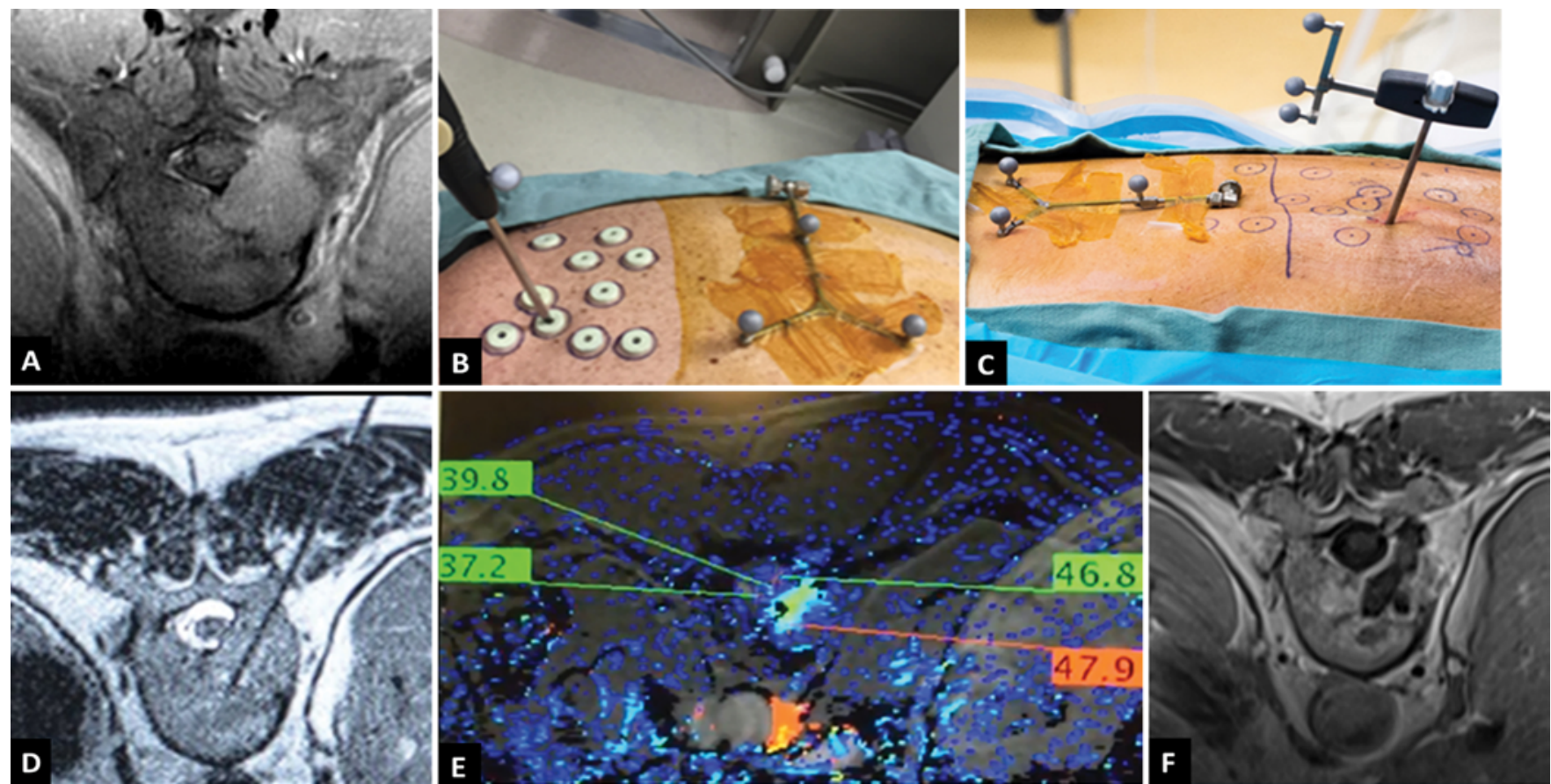

FIG. 1. Overview of the SLITT procedure. A: Preoperative axial MR image demonstrating metastatic cholangiocarcinoma causing ESCC grade 1c with symptomatic T10 radiculopathy. B: MRI fiducials are applied dorsally. Intraoperative MRI is performed, and surface-matching registration is performed for image guidance. C: A navigated Jamshidi needle is inserted into the tumor and exchanged to an MRI-compatible access cannula (not shown). D: Intraoperative MR image localizing the laser fiber prior to the ablation. E: Screenshot of intraoperative MRI thermography during the laser ablation. Green boxes show the temperatures in the interface of the dura/tumor, and automatic shutoff is programmed once temperature reaches $48^{\circ} \mathrm{C}-50^{\circ} \mathrm{C}$. F: Postoperative axial MR image demonstrating reconstitution of the thecal sac and local control of the epidural tumor at 4 months post-SLITT+SSRS.

which allows placement of the laser catheter (Visualase, Medtronic, Inc.) (Fig. 1).

The laser ablation is performed with real-time iMRI thermography under ventilator pause as previously described. ${ }^{17}$ If stabilization is required, the patient is removed from the iMRI unit and positioned at a safe distance from the high magnetic field where percutaneous instrumentation can be inserted using either MRI guidance or standard fluoroscopic technique. ${ }^{13}$ In cases of severe osteoporosis, we perform methylmethacrylate augmentation of the screws ${ }^{14}$ to increase the purchase in bone, aiming to achieve a more durable stabilization. Follow-up imaging is performed generally $6-12$ weeks after SLITT.

\section{Imaging and Follow-Up}

All patients underwent postoperative MRI and were evaluated as outpatients approximately every 3-4 months. The radiation oncology team, in the context of tumor board recommendations, made treatment decisions regarding postoperative radiotherapy (either cEBRT or SSRS), including timing, modality, and dosimetry. In general, in patients who underwent SLITT and were referenced for SSRS, the procedure was planned and delivered based on the preoperative MRI findings. The treatment plan was calculated as if no ablation had been performed, maximally covering the gross target volume and limiting spinal cord exposure to $12 \mathrm{~Gy}$. Similarly, treatment decisions regarding postoperative chemotherapy were made at the discretion of the treating medical oncologist. To monitor for tumor recurrence, patients underwent serial MRI generally at 3- to 4-month intervals.

The degree of spinal cord compression before and after open surgery or SLITT was scored according to the validated 6-point ESCC scale. ${ }^{2}$ Any increase in the ESCC score from the initial postprocedure MRI scan was considered radiographic failure. Examples of local control are provided in Fig. 2, and examples of treatment failure are demonstrated in Fig. 3.

\section{Statistical Analysis}

A Mann-Whitney U-test was used to compare EBL, LOS, and time to chemotherapy and radiotherapy between the two treatment groups. Proportions and clinical characteristics were examined using Pearson chi-square analysis. For contingency table analysis with multiple degrees of freedom, we utilized a post hoc analysis with the appropriate Bonferroni correction. ${ }^{1}$ A logistic regression was created to analyze predictive factors for complications, including age, sex, KPS score, ESCC score, and treatment (SLITT vs open surgery).

OS and PFS curves were estimated using Kaplan-Meier analysis comparing SLITT to open surgery. To evaluate predictors of local recurrence, a univariate analysis using the Enter method of binary logistics was created for each variable. Variables with $\mathrm{p}<0.25$ in the univariate analysis were included in a Cox model using forward stepwise method. Statistical significance was determined as $\mathrm{p}<$ 

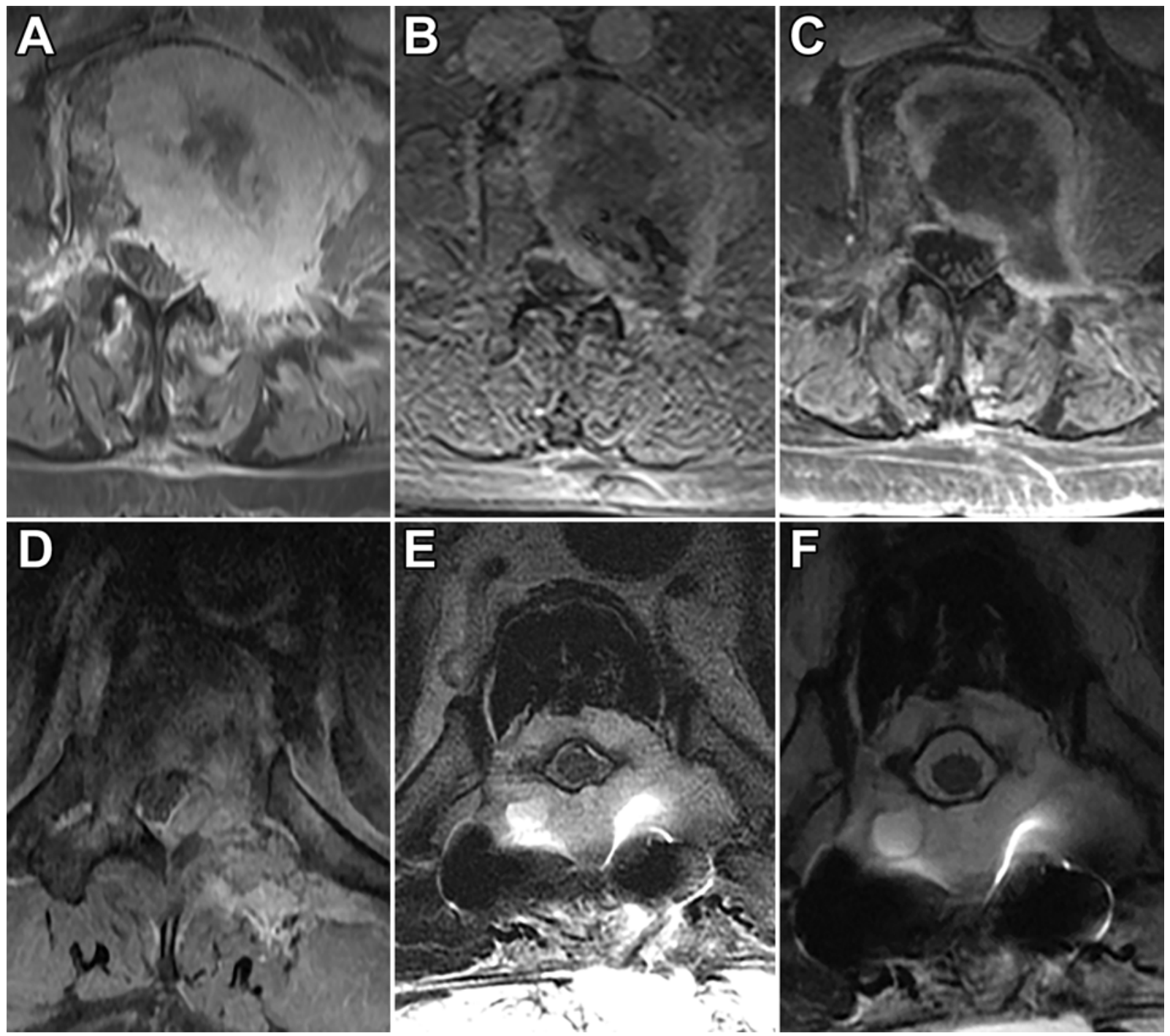

FIG. 2. Axial MR images showing successful local control after SLITT (upper) and open surgery (lower). A: Preoperative image obtained in a patient treated who underwent SLITT, demonstrating metastatic renal cell carcinoma involving the L3 vertebral body. B: Image obtained immediately postoperatively (prior to SSRS), demonstrating a decrease in the tumor enhancement after SLITT.

C: Image obtained at the 12-month follow-up, demonstrating local control with decrease in the baseline epidural compression.

D: Preoperative image obtained in a patient who underwent open surgery, demonstrating metastatic renal cell carcinoma involving the T10 vertebral body. E: Image obtained immediately postoperatively (prior to SSRS) demonstrating complete resection of the epidural tumor. F: Image obtained at the 12-month follow-up, demonstrating local control with resolution of the mass effect.

0.05. Statistical analysis was performed using IBM SPSS (version 24, IBM Corp.).

\section{Results \\ Patient Characteristics}

Eighty patients were included in this analysis, with 40 patients in each group. Patient demographics, spinal levels, preprocedure ESCC scores, and clinical features are demonstrated in (Table 1). The most prevalent tumor histology treated was renal cell carcinoma, followed by non-small cell lung cancer (Table 2).

\section{Postprocedure Course and Adjuvant Treatment}

Table 3 describes clinical information of the patients after the procedure. Morbidity was higher in the group treated with open surgery than in the group treated with SLITT. Fourteen patients (35\%) treated with open surgery presented with postoperative complications compared with 2 patients $(5 \%)$ treated with SLITT $(\mathrm{p}=0.003)$. When analyzing complications in general (minor and major), patients in the open surgery group had an HR of 11.588 (95\% CI $2.281-58.865, \mathrm{p}=0.003$ ). When analyzing only major complications, patients in the open surgery group had an HR of 16.020 (95\% CI 1.814-141.506, $\mathrm{p}=0.013)$ when compared with the SLITT group. For a full description of complications, refer to Supplemental Table 1.

Open surgery had a significant advantage in terms of complete decompression of the spinal canal. Comparison of the preprocedure and the initial postprocedure MRI 

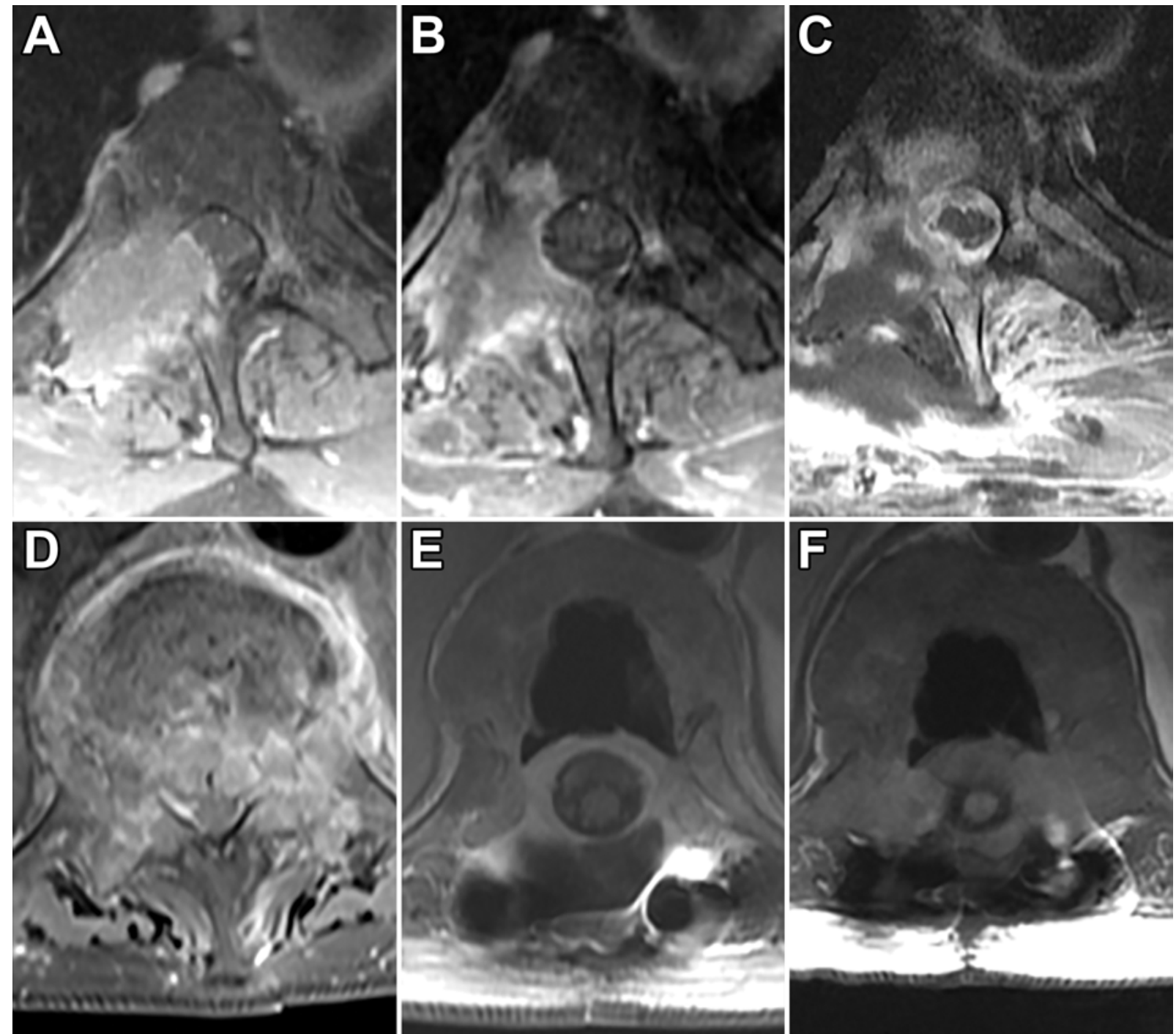

FIG. 3. Axial MR images showing failure of local control after SLITT (upper) and open surgery (lower). A: Preoperative image obtained in a patient treated with SLITT, demonstrating metastatic renal cell carcinoma involving the T4 dorsal elements. B: Image obtained at the 2-month follow-up after SLITT and SSRS, demonstrating decrease in tumor enhancement and resolution of epidural compression. C: Image obtained at the 9-month follow-up, demonstrating local failure. D: Preoperative image obtained in a patient treated with vertebrectomy, demonstrating metastatic colon cancer involving the T11 vertebral body. E: Image obtained at the 2-month follow-up after vertebrectomy and SSRS, demonstrating decrease in the tumor enhancement and resolution of epidural compression. F: Image obtained at the 9-month follow-up, demonstrating local failure.

scans demonstrated that more patients who underwent open surgery had ESCC scores that decreased to 0 than those who underwent SLITT ( $p<0.001$ ). However, 72.5\% of cases treated with SLITT did experience a reduction in ESCC score. The median preoperative ESCC score of 2 reduced to $1 \mathrm{~b}$ after SLITT, whereas after open surgery the median ESCC sore went from 2 to no compression. All patients underwent adjuvant radiosurgery. The majority of patients in the SLITT group underwent adjuvant SSRS (97.5\%), whereas $55 \%$ of cases in the open surgery group had adjuvant cEBRT $(\mathrm{p}<0.001)$. In the open surgery group $92.5 \%$ of patients underwent spinal instrumentation, compared with $15 \%$ in the SLITT group ( $<<0.001$; Table 3 ).

Table 4 shows the differences in postprocedure variables between the two groups. The SLITT group had a substantially lower blood loss with a mean of $117 \mathrm{ml}$ (SD $84 \mathrm{ml})$ compared with a mean of $1331 \mathrm{ml}(\mathrm{SD} 1219 \mathrm{ml})$ recorded in the open surgery group $(\mathrm{U}=56.5, \mathrm{p}<0.001)$. As expected with a less invasive procedure, the mean hospital LOS of patients treated with SLITT was 3.4 days (SD 4.3 days), significantly lower than 9 days (SD 5.5 days) in patients treated with open surgery $(U=244, p<0.001)$. Patients in the SLITT group had a shorter time between the procedure and the initiation of radiotherapy with a mean time of 7.8 days (SD 11.8 days) compared with 35.9 days (SD 32.7 days) in the open surgery group ( $U=154$, $p$ $<0.001)$. Similarly, the mean time to begin postprocedure systemic therapy was 24.7 days (SD 21 days) in the SLITT group, significantly shorter than that in the open surgery group (59 days, $\mathrm{SD} 80$ days, $\mathrm{U}=68, \mathrm{p}=0.015$ ) (Table 4). 
TABLE 1. Patient demographic, clinical, systemic, and local therapy information at diagnosis of spine metastasis stratified by subtype

\begin{tabular}{|c|c|c|c|}
\hline Type & SLITT $(\%)$ & Open Surgery (\%) & $p$ Value \\
\hline Age, yrs & & & 0.212 \\
\hline$<50$ & $7(17.5)$ & $6(15)$ & \\
\hline $51-60$ & $11(27.5)$ & $20(50)$ & \\
\hline $61-70$ & $15(37.5)$ & $10(25)$ & \\
\hline$>71$ & $7(17.5)$ & $4(10)$ & \\
\hline Sex & & & 0.793 \\
\hline Male & $30(75)$ & $31(77.5)$ & \\
\hline Female & $10(25)$ & $9(22.5)$ & \\
\hline ESCC score preprocedure & & & 0.621 \\
\hline 1c & $14(35)$ & $10(25)$ & \\
\hline 2 & $13(32.5)$ & $15(37.5)$ & \\
\hline 3 & $13(32.5)$ & $15(37.5)$ & \\
\hline Prior treatment & & & 0.457 \\
\hline None & $27(67.5)$ & $25(62.5)$ & \\
\hline cEBRT & $8(20)$ & $13(32.5)$ & \\
\hline SSRS & $2(5)$ & $1(2.5)$ & \\
\hline cEBRT \& SSRS & $3(7.5)$ & $1(2.5)$ & \\
\hline KPS score & & & 0.329 \\
\hline$>70$ & $26(65)$ & $30(75)$ & \\
\hline$<70$ & $14(35)$ & $10(25)$ & \\
\hline Multiple levels & & & 0.639 \\
\hline Yes & $25(62.5)$ & $27(67.5)$ & \\
\hline No & $15(37.5)$ & $13(32.5)$ & \\
\hline Total & $40(100)$ & $40(100)$ & \\
\hline
\end{tabular}

TABLE 2. Tumor histology before SLITT or open surgery*

\begin{tabular}{lcc}
\hline Histology & SLITT (\%) & Open Surgery (\%) \\
\hline RCC & $17(42.5)$ & $11(27.5)$ \\
NSCLC & $5(12.5)$ & $6(15)$ \\
HCC & $3(7.5)$ & $3(7.5)$ \\
Colon & $0(0)$ & $4(10)$ \\
Breast & $0(0)$ & $2(5)$ \\
Melanoma & $3(7.5)$ & $0(0)$ \\
Sarcoma & $3(7.5)$ & $4(10)$ \\
Thyroid & $3(7.5)$ & $4(10)$ \\
Other† & $6(15)$ & $6(15)$ \\
Total & $40(100)$ & $40(100)$
\end{tabular}

$\mathrm{HCC}=$ hepatocellular carcinoma; NSCLC = non-small cell lung cancer $\mathrm{RCC}=$ renal cell carcinoma.

* There were no statistically significant differences in the overall model between the groups in regard to histology (chi-square, $p=0.222$ ).

† Cholangiocarcinoma, chordoma, hemangiopericytoma, leiomyosarcoma, liposarcoma, neuroendocrine tumor, osteosarcoma, parotid carcinoma, pheochromocytoma, rectal carcinoma, synovial sarcoma, squamous cell carcinoma, thyroid follicular carcinoma, thyroid medullary carcinoma, thyroid Hürthle cell carcinoma, thyroid papillary carcinoma, and uterine adenocarcinoma.
TABLE 3. Patient's clinical information after SLITT or open surgery

\begin{tabular}{lccc}
\hline \multicolumn{1}{c}{ Type } & SLITT (\%) & Open Surgery (\%) & p Value \\
\hline Complications & & & \\
\hline Overall & $2(5)$ & $14(35)$ & 0.003 \\
\hline Minor & $1(2.5)$ & $4(10)$ & $0.003^{*}$ \\
\hline Major & $1(2.5)$ & $10(25)$ & $<0.001^{*}$ \\
\hline None & $38(95)$ & $26(65)$ & $<0.160$ \\
\hline Adjuvant radiotherapy & & & $<0.001$ \\
\hline SSRS & $39(97.5)$ & $18(45)$ & \\
\hline cEBRT & $1(2.5)$ & $22(55)$ & \\
\hline Spinal instrumentation & & & $<0.001$ \\
\hline Yes & $6(15)$ & $37(92.5)$ & \\
\hline No & $34(85)$ & $3(7.5)$ & \\
\hline Reduction in ESCC score & & & $<0.045$ \\
\hline Yes & $29(72.5)$ & $36(90)$ & \\
\hline No & $11(27.5)$ & $4(10)$ & \\
\hline ESCC score postprocedure & & & \\
\hline 0 & $5(12.5)$ & $22(55)$ & $<0.001^{*}$ \\
\hline 1a & $7(17.5)$ & $5(12.5)$ & 0.130 \\
\hline 1b & $9(22.5)$ & $4(10)$ & 0.075 \\
\hline 1c & $7(17.5)$ & $2(5)$ & 0.163 \\
\hline 2 & $4(10)$ & $1(2.5)$ & 0.163 \\
\hline 3 & $7(17.5)$ & $3(7.5)$ & 0.320 \\
\hline Total & $40(100)$ & $40(100)$ & \\
\hline Bolface & &
\end{tabular}

Boldface type indicates statistical significance.

* Statistically significant after the appropriate Bonferroni correction.

\section{Outcomes}

The median follow-up time was 13 months (95\% CI 9-16 months) for all patients. There was no overall difference in PFS between patients who underwent open surgery (median 24 months, 95\% CI 1.5-46.4 months) and SLITT (median not reached, $\mathrm{p}=0.510$ ) (Fig. 4). A comparison between patients receiving only SSRS in either group did not show a significant difference $(\mathrm{p}=0.605$; Supplemental Fig. 1). The local control rates at 12 months and 24 months were $71.2 \%$ (SE 8.8\%) and 64\% (SE 10.4\%) in the SLITT group and 78.5\% (SE 7.4\%) and 55.2\% (SE 11.5\%) in the open surgery group $(\mathrm{p}=0.510)$. There was no difference in median OS between SLITT and open surgery; patients

TABLE 4. Comparison of variable means between SLITT and open surgery groups

\begin{tabular}{lccr}
\hline \multicolumn{1}{c}{ Type } & SLITT (SD) & Open Surgery (SD) & p Value \\
\hline Mean EBL, ml & $117(84)$ & $1331(1219)$ & $<0.001$ \\
Mean LOS, days & $3.4(4.3)$ & $9(5.5)$ & $<0.001$ \\
Mean time to systemic & $24.7(21)$ & $59(80)$ & 0.015 \\
$\quad$ therapy, days & & & \\
$\begin{array}{l}\text { Mean time to SSRS or } \\
\text { cEBRT, days }\end{array}$ & $7.8(11.8)$ & $35.9(32.7)$ & $<0.001$ \\
\hline
\end{tabular}

Boldface type indicates statistical significance. 


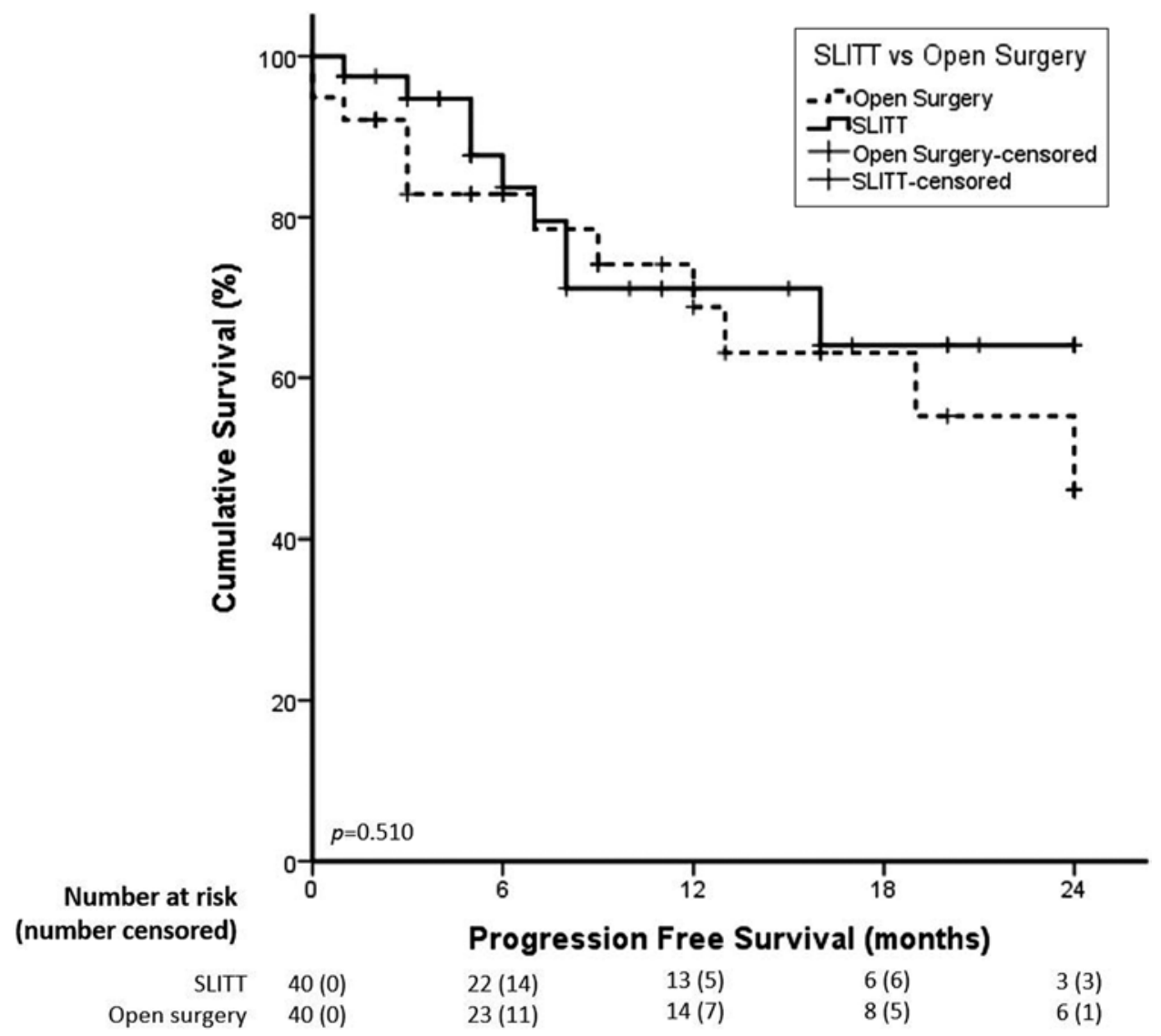

FIG. 4. Kaplan-Meier estimates of PFS comparing SLITT and open surgery cohorts only with patients who underwent adjuvant SSRS.

treated with SLITT had a median OS of 12 months (95\% CI 6.7-17.3 months) compared with 13 months (95\% CI $8.4-17.5$ months) in the patients treated with open surgery $(\mathrm{p}=0.868)$.

On the univariate analysis for local recurrence in the open surgery group, an ESCC score preprocedure $\geq 2$ and procedure in multiple levels were statistically significant $(p=0.040$ and $p=0.018$, respectively). KPS score, ESCC score postprocedure, and primary tumor histology did not reach statistical significance. On the multivariate Cox proportional hazard model that included the statistically significant variables, multiple levels procedure was the only independent predictive factor for local recurrence identified with an HR of 3.922 (95\% CI 1.160-13.265, p $=0.028)$. In the SLITT group, no predictors of local recurrence could be identified in the uni- and multivariate models.

\section{Discussion}

We report a retrospective comparison of outcomes in a matched cohort of patients with epidural metastatic disease treated with distinct modalities. We selected cases that could be treated with either open surgery or SLITT. There was some variability in the open surgery group, such as the extent of surgery in terms of performance of anterior reconstruction and the number of spinal levels instrumented; however, we believe that similar variability would occur in the SLITT group, provided they were managed with open surgery. Our intention was to illustrate the potential use of SLITT as an alternative to open surgery in very-well-selected cases, as this analysis demonstrated that SLITT is associated with lower EBL, shorter LOS, fewer complications, shorter time to adjuvant radiotherapy, and shorter delays to reinitiation of systemic therapy than open surgery. The ideal candidate for SLITT is a patient with metastatic ESCC between T2 and T12, who is neurologically intact or with minor sensory symptoms, where a separation surgery is required before SSRS, particularly for those with progressive systemic disease and significant comorbidities that would increase the risk of open surgery ${ }^{15}$ Patients with cervical or lumbar metastatic ESCCs are not candidates for SLITT due to a higher risk of complications. In addition, patients with neurological deficits requiring urgent decompression are not candidates for SLITT, since the procedure does not confer immediate epidural decompression.

The utilization of cEBRT in radiosensitive histologies and SSRS as a primary treatment modality for radioresistant tumors can provide durable local control of spinal metastasis. When SSRS is considered, dosimetric studies have indicated that approximately $2 \mathrm{~mm}$ of separation between the tumor and spinal cord is required for adequate tumoricidal dosing targeting the gross tumor volume 
while respecting the radiation constraints of the spinal cord. ${ }^{11}$ Underdosing the lesion at the tumor-dura interface risks epidural failure where it is most likely to result in spinal cord compression and neurological compromise. Open surgical decompression is traditionally used to create the required spatial separation, and we believe that selected cases can benefit from SLITT as it could offer improved risk-benefit ratio facilitating a faster recovery and return to the intended oncological treatment.

Epidural decompression achieved with SLITT is not immediate and occurs over weeks after the procedure; therefore, patients with acute neurological deficits are better served with an immediate decompression achieved with open surgery. ${ }^{18}$ Patients treated with SLITT presented with a lower average decrease in ESCC score than patients treated with open surgery; nonetheless, ablation of the epidural component was sufficient to allow adequate radiation coverage to the entire tumor site. The analysis of PFS between patients treated with SLITT and open surgery followed by adjuvant radiotherapy did not differ between groups. We recognize that more patients were treated with cEBRT than SSRS in the open surgery group; therefore, the analysis of local control with open surgery can be subject to bias, as local recurrence rates achieved with cEBRT and SSRS can be different. However, a comparison between patients receiving only SSRS in either group did not show a statistically significant difference, supporting the notion that SLITT can provide local control similar to that of open surgery when followed by SSRS. As for predictive factors for local recurrence, only metastatic disease affecting multiple contiguous spinal levels was considered to increase local treatment failure in the open surgery group.

The less invasive nature of SLITT was reflected by the substantially lower complication rates when compared with open surgery. We found the complication rate for the SLITT group to be equivalent to what we have previously reported. ${ }^{4,9}$ Additionally, hospital length of stay and blood loss were also reduced in the SLITT group. This is particularly important in a population often already weakened by the burden of advanced disease. We also demonstrate that these patients were able to resume chemotherapy, as well as start adjuvant radiotherapy, sooner in comparison with the open surgery group. Another important difference to highlight is the need for spinal instrumentation after epidural decompression between both groups. In this study, all open cases were performed through a posterior approach, which was often associated with significant disruption of the dorsal elements, creating the need for placement of pedicle screws and, in some cases, anterior column reconstruction. SLITT does not disrupt the posterior elements (when intact preprocedure) and does not necessarily result in spinal instability.

This study was subject to the many well-known limitations of retrospective studies. The cohorts were not individually matched, but the groups were matched to reflect a homogeneous sample. As the magnitude of the open surgery and the type of adjuvant radiation was not controlled, the open surgical group does not represent separation surgery as proposed by Bilsky et al. ${ }^{2}$ with which SLITT should ideally be compared. Although we performed a retrospective analysis of the preoperative images and includ- ed patients who could be treated by either modality, a selection bias for patients with a higher degree of spinal cord compression to be treated with open surgery and patients with more significant comorbidities to be treated with SLITT could be a potential issue. We also recognize a significant discrepancy in the type of adjuvant radiotherapy. The fact that open resection significantly eliminated more of the local tumor in combination with a longer time for wound healing may explain the delay in the recommendation for adjuvant radiotherapy postoperatively. Additionally, patients presented in vastly different stages of their disease course, having undergone a variety of local and systemic treatments. The sum of these potentially offsetting differences are unknown. The groups in this study do, however, represent a "real-world" cross-section of patients presenting to a tertiary cancer center over a given time.

\section{Conclusions}

This report provides further rationale for the exploration of SLITT as another treatment modality to join the armamentarium against spinal metastatic disease. The technique appears to be well tolerated with a lower rate of complications and can be of great value to a broader set of patients who would not normally be considered good candidates for open surgery. Our results have shown that SLITT plus radiotherapy is not inferior to open decompression surgery plus radiotherapy in regard to local control. We believe that the results presented in this article provide justification for a prospective randomized study to compare open surgical decompression and SLITT followed by SSRS in the management of high-grade ESCC.

\section{References}

1. Beasley TM, Schumacker RE: Multiple regression approach to analyzing contingency tables: post hoc and planned comparison procedures. J Exp Educ 64:79-93, 1995

2. Bilsky MH, Laufer I, Fourney DR, Groff M, Schmidt MH, Varga PP, et al: Reliability analysis of the epidural spinal cord compression scale. J Neurosurg Spine 13:324-328, 2010

3. Bishop AJ, Tao R, Rebueno NC, Christensen EN, Allen PK, Wang XA, et al: Outcomes for spine stereotactic body radiation therapy and an analysis of predictors of local recurrence. Int J Radiat Oncol Biol Phys 92:1016-1026, 2015

4. Choi D, Fox Z, Albert T, Arts M, Balabaud L, Bunger C, et al: Rapid improvements in pain and quality of life are sustained after surgery for spinal metastases in a large prospective cohort. Br J Neurosurg 30:337-344, 2016

5. Frankel HL, Hancock DO, Hyslop G, Melzak J, Michaelis LS, Ungar GH, et al: The value of postural reduction in the initial management of closed injuries of the spine with paraplegia and tetraplegia. I. Paraplegia 7:179-192, 1969

6. Gerszten PC: Spine metastases: from radiotherapy, surgery, to radiosurgery. Neurosurgery 61 (Suppl 1): 16-25, 2014

7. Ghia AJ, Chang EL, Bishop AJ, Pan HY, Boehling NS, Amini B, et al: Single-fraction versus multifraction spinal stereotactic radiosurgery for spinal metastases from renal cell carcinoma: secondary analysis of Phase I/II trials. J Neurosurg Spine 24:829-836, 2016

8. Ghia AJ, Rebueno NC, Li J, Brown PD, Rhines LD, Tatsui CE: The use of image guided laser interstitial thermotherapy to supplement spine stereotactic radiosurgery to manage metastatic epidural spinal cord compression: proof of concept 
and dosimetric analysis. Pract Radiat Oncol 6:e35-e38, 2016

9. Gokaslan ZL, York JE, Walsh GL, McCutcheon IE, Lang FF, Putnam JB Jr, et al: Transthoracic vertebrectomy for metastatic spinal tumors. J Neurosurg 89:599-609, 1998

10. Laufer I, Iorgulescu JB, Chapman T, Lis E, Shi W, Zhang Z, et al: Local disease control for spinal metastases following "separation surgery" and adjuvant hypofractionated or highdose single-fraction stereotactic radiosurgery: outcome analysis in 186 patients. J Neurosurg Spine 18:207-214, 2013

11. Moussazadeh N, Laufer I, Yamada Y, Bilsky MH: Separation surgery for spinal metastases: effect of spinal radiosurgery on surgical treatment goals. Cancer Contr 21:168-174, 2014

12. Patchell RA, Tibbs PA, Regine WF, Payne R, Saris S, Kryscio RJ, et al: Direct decompressive surgical resection in the treatment of spinal cord compression caused by metastatic cancer: a randomised trial. Lancet 366:643-648, 2005

13. Silbergleit R, Mehta BA, Sanders WP, Talati SJ: Imagingguided injection techniques with fluoroscopy and CT for spinal pain management. Radiographics 21:927-942, 2001

14. Tatsui CE, Belsuzarri TA, Oro M, Rhines LD, Li J, Ghia AJ, et al: Percutaneous surgery for treatment of epidural spinal cord compression and spinal instability: technical note. Neurosurg Focus 41(4):E2, 2016

15. Tatsui CE, Lee SH, Amini B, Rao G, Suki D, Oro M, et al: Spinal laser interstitial thermal therapy: a novel alternative to surgery for metastatic epidural spinal cord compression. Neurosurgery 79 (Suppl 1):S73-S82, 2016

16. Tatsui CE, Nascimento CNG, Suki D, Amini B, Li J, Ghia AJ, et al: Image guidance based on MRI for spinal interstitial laser thermotherapy: technical aspects and accuracy. J Neurosurg Spine 26:605-612, 2017

17. Tatsui CE, Stafford RJ, Li J, Sellin JN, Amini B, Rao G, et al: Utilization of laser interstitial thermotherapy guided by real-time thermal MRI as an alternative to separation surgery in the management of spinal metastasis. J Neurosurg Spine 23:400-411, 2015

18. Thomas JG, Al-Holou WN, de Almeida Bastos DC, Ghia AJ, Li J, Bishop AJ, et al: A novel use of the intraoperative MRI for metastatic spine tumors: laser interstitial thermal therapy for percutaneous treatment of epidural metastatic spine disease. Neurosurg Clin N Am 28:513-524, 2017

19. Vitaz TW, Oishi M, Welch WC, Gerszten PC, Disa JJ, Bilsky $\mathrm{MH}$ : Rotational and transpositional flaps for the treatment of spinal wound dehiscence and infections in patient popula- tions with degenerative and oncological disease. J Neurosurg 100 (1 Suppl Spine):46-51, 2004

20. Yamada Y, Bilsky MH, Lovelock DM, Venkatraman ES, Toner S, Johnson J, et al: High-dose, single-fraction imageguided intensity-modulated radiotherapy for metastatic spinal lesions. Int J Radiat Oncol Biol Phys 71:484-490, 2008

21. Zacharia BE, Kahn S, Bander ED, Cederquist GY, Cope WP, McLaughlin L, et al: Incidence and risk factors for preoperative deep venous thrombosis in 314 consecutive patients undergoing surgery for spinal metastasis. J Neurosurg Spine 27:189-197, 2017

\section{Disclosures}

The authors report no conflict of interest concerning the materials or methods used in this study or the findings specified in this paper.

\section{Author Contributions}

Conception and design: Tatsui, Bastos, Everson, Rao, Li, Ghia, Bishop, Yeboa, Amini, Rhines. Acquisition of data: Tatsui, Bastos, Everson, Habib. Analysis and interpretation of data: Tatsui, Bastos, Everson, Santos. Drafting the article: Tatsui, Bastos, Everson. Critically revising the article: Tatsui, Bastos, Everson, Vega, Li, Ghia, Bishop, Yeboa, Amini, Rhines. Reviewed submitted version of manuscript: Tatsui, Bastos, Everson, Vega, Rao, Li, Bishop, Yeboa, Amini, Rhines. Approved the final version of the manuscript on behalf of all authors: Tatsui. Statistical analysis: Tatsui, Bastos, Everson. Administrative/ technical/material support: Tatsui, Bastos, Oro. Study supervision: Tatsui, Bastos, Everson, Oro, Rao.

\section{Supplemental Information}

\section{Online-Only Content}

Supplemental material is available with the online version of the article.

Supplemental Table 1 and Fig. 1. https://thejns.org/doi/ suppl/10.3171/2019.10.SPINE19998.

\section{Correspondence}

Claudio Esteves Tatsui: The University of Texas MD Anderson Cancer Center, Houston, TX. cetatsui@mdanderson.org. 